\title{
REVIEW
}

\section{Genetics of Paget's disease of bone}

\author{
Omar ME Albagha
}

Centre for Genomic and Experimental Medicine, Institute of Genetics and Molecular Medicine, University of Edinburgh, Edinburgh, UK.

Paget's disease of bone (PDB) is a common metabolic bone disease characterised by focal areas of increased bone turnover, which primarily affects people over the age of 55 years. Genetic factors have a fundamental role in the pathogenesis of PDB and are probably the main predisposing factor for the disease. The genetic contribution to PDB susceptibility ranges from rare pathogenic mutations in the single gene SQSTM1 to more common, small effect variants in at least seven genetic loci that predispose to the disease. These loci have additive effects on disease susceptibility and interact with SQSTM1 mutations to affect disease severity, making them a potentially useful tool in predicting disease risk and complication and in managing treatments. Many of these loci harbour genes that have important function in osteoclast differentiation such as CSF1, DCSTAMP and TNFRSF11A. Other susceptibility loci have highlighted new molecular pathways that have not been previously implicated in regulation of bone metabolism such as OPTN, which was recently found to negatively regulate osteoclast differentiation. PDB-susceptibility variants exert their effect either by affecting the protein coding sequence such as variants found in SQSTM1 and RIN3 or by influencing gene expression such as those found in OPTN and DCSTAMP. Epidemiological studies indicate that environmental triggers also have a key role in PDB and interact with genetic factors to influence manifestation and severity of the disease; however, further studies are needed to identify these triggers.

BoneKEy Reports 4, Article number: 756 (2015) | doi:10.1038/bonekey.2015.125

\section{Introduction}

Paget's disease of bone (PDB) is a common skeletal disorder characterised by focal areas of increased and disorganised bone turnover, which primarily target the axial skeleton. Other abnormalities include bone marrow fibrosis and increased vascularity of affected bones. The most common symptom is bone pain, but other complications may also occur including bone deformity, deafness, osteoarthritis and fracture. In some cases, the disease can be severely disabling, leading to a substantial reduction in the quality of life. PDB is a late-onset disease that rarely affects people below the age of 55 years. The prevalence of the disease is about $1 \%$ in people of European descent but increases significantly with age to affect about $5 \%$ of people aged 85 years and over in some countries. ${ }^{1,2}$ At a cellular level, osteoclasts from affected bone are more abundant, appear larger in size, contain many more nuclei per cell and have increased resorption activity compared with normal osteoclasts. There is also an accompanied marked increases in osteoblast activity resulting in the formation of disorganised 'woven' bone, which has reduced mechanical strength, leading to increased risk of fracture.
The past decade has witnessed major advances in our understanding of the mechanisms by which genetic factors predispose to Paget's disease. This article reviews these advances and reflects on the mechanisms by which genetic factors interact with environmental triggers to influence the development of PDB.

\section{Genetic Factors and PDB}

The contribution of genetic factors to PDB was first reported in 1949, and since then accumulated evidence shows that genetic factors have a key role in pathogenesis. Familial clustering is common in PDB, and in many families the disease follows an autosomal dominant mode of inheritance with incomplete penetrance. Patients rarely show symptoms of the disease before the age of 50 years, but the penetrance of the disease increases with age to reach almost $90 \%$ by the age of 80 years. ${ }^{3-6}$ About $15 \%$ of patients report a positive family history, but the proportion of familial cases may actually be higher because the disease is often asymptomatic. Relatives of an affected person have approximately seven times greater risk of developing PDB than relatives of controls, and this risk increases to about 20 times for relatives of patients with severe 
and early-onset disease. ${ }^{7}$ PDB cases without reported family history are termed 'sporadic' in which the disease could occur as a result of isolated de novo mutations, environmental triggers or simply due to incomplete penetrance obscuring family history. The prevalence of PDB varies widely in different ethnic groups. People of Western European descent are most commonly affected, and the highest prevalence is in the UK. ${ }^{8}$ The disease is uncommon in Scandinavia and rare in India, China, Japan and other far eastern countries. These ethnic variations in disease prevalence persist after migration from high prevalence regions such as UK and Europe to countries like Australia, New Zealand and Canada where PDB is rare in the native population. This could be explained by the occurrence of old 'founder' mutations or polymorphisms that predispose to the disease in people from Europe who then spread to other parts of the world via emigration. ${ }^{9,10}$ Several candidate genes and genomic loci have been associated or linked to PDB, ${ }^{4,5,11}$ confirming the contribution of genetic factors to the disease aetiology (Table 1). Many of the PDB-associated genes have important roles in osteoclast differentiation and/or function, and many cluster in the RANKL-OPG-RANK signalling pathway (Figure 1). These genetic loci are the main focus of this article, and they are described in details below.

\section{SQSTM1 Mutations in Classical PDB}

Several genetic loci have been identified using the genome-wide linkage scan approach, which include susceptibility loci for familial PDB on chromosome $5 q 31,5 q 35$ and 10p13., ${ }^{4,5}$ The strongest evidence for linkage was reported for the $5 \mathrm{q} 35$ locus, which was confirmed by two independent studies $^{4,5}$, and subsequent positional cloning studies showed that mutations in SQSTM1 gene are responsible for $5 q 35$-linked PDB. ${ }^{12,13}$ SQSTM1 encodes p62, which is an adaptor protein that binds ubiquitin and has an important role in the NFKB signalling pathway. ${ }^{14}$ Several SQSTM1 mutations have been reported in PDB patients, and most affect the ubiquitin-associated domain (UBA) of p62. Mutations in
SQSTM1 occur in about $40 \%$ of familial PDB cases and up to $10 \%$ of sporadic cases. ${ }^{6,15}$ The mechanism by which mutations in SQSTM1 gene lead to PDB development is still unclear, but existing evidence suggests that mutations in the UBA domain interfere with the ability of p62 to bind ubiquitin leading to

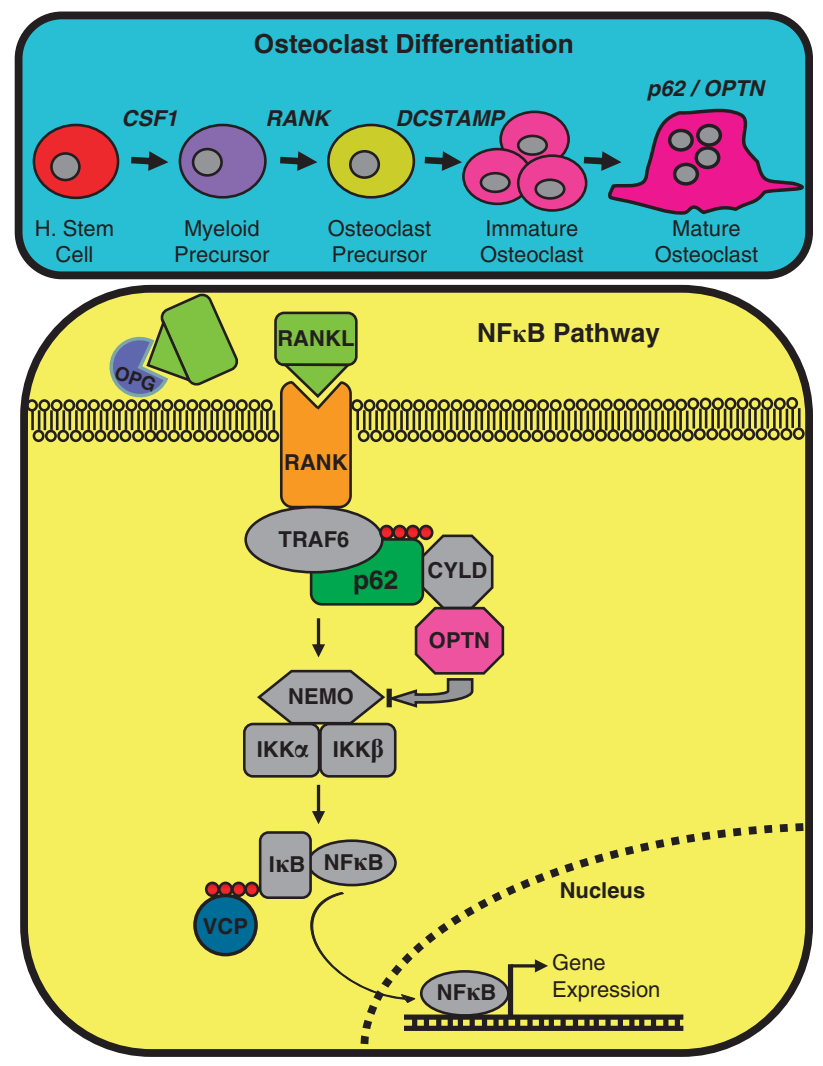

Figure 1 Genes that predispose to PDB are implicated in the osteoclast differentiation (top panel) and the RANKL-OPG-RANK signalling pathways (bottom panel).

Table 1 Genes and genetic loci implicated in the pathogenesis of Paget's disease and related syndromes

\begin{tabular}{|c|c|c|c|c|}
\hline Locus & Gene(s) & Protein & Function & Phenotype \\
\hline $5 q 35$ & SQSTM1 & p62 & Adaptor protein involved in NFKB signalling pathway & $\begin{array}{l}\text { Mutations cause } 10 \% \text { of sporadic and } 40 \% \text { of } \\
\text { familial cases of classical PDB }\end{array}$ \\
\hline $8 q 22$ & $\begin{array}{l}\text { DCSTAMP } \\
\text { (TM7SF4) }\end{array}$ & DCSTAMP & Role in fusion of osteoclast precursors & $\begin{array}{l}\text { Common variants predispose to classical PDB } \\
\text { and regulate DCSTAMP expression }\end{array}$ \\
\hline $9 p 21$ & $V C P$ & VCP & $\begin{array}{l}\text { Involved in proteosomal degradation of } \\
\text { ubiqutinated proteins and in vesicular trafficking }\end{array}$ & $\begin{array}{l}\text { Mutations cause Inclusion body myopathy } \\
\text { Paget's disease with frontotemporal dementia }\end{array}$ \\
\hline $10 p 13$ & OPTN & Optineurin & $\begin{array}{l}\text { Negative regulation of osteoclast differentiation by } \\
\text { modulation of } N F_{K} B \text { and IFN- } \beta \text { signalling pathways }\end{array}$ & $\begin{array}{l}\text { Common variants predispose to classical PDB } \\
\text { by regulating OPTN expression }\end{array}$ \\
\hline $14 q 32$ & RIN3 & $\begin{array}{l}\text { Ras rab } \\
\text { interactor } 3\end{array}$ & $\begin{array}{l}\text { Unknown role in bone metabolism, could be } \\
\text { involved in autophagy }\end{array}$ & $\begin{array}{l}\text { Common and rare missense variants predispose } \\
\text { to classical PDB }\end{array}$ \\
\hline $15 q 24$ & $P M L / G O L G A 6 A$ & PML/GOLGA6 & $\begin{array}{l}\text { Unknown role in bone metabolism, involved in } \\
\text { apoptosis and TGF- } \beta \text { signalling }\end{array}$ & Common variants predispose to classical PDB \\
\hline
\end{tabular}

Abbreviations: DCSTAMP, dendritic cells-specific transmembrane protein; INF- $\beta$, interferon- $\beta$; M-CSF, macrophage-colony stimulating factor; PDB, Paget's disease of bone; PML, promyelocytic leukaemia; TGF- $\beta$, transforming growth factor- $\beta$. 
enhanced NFKB signalling and increased sensitivity of osteoclasts precursors to RANKL. ${ }^{16,17}$

SQSTM1 is the only known gene that is mutated in the classical form of PDB. Investigation of other genomic regions linked to PDB (5q31 and 10p13) by positional cloning has so far failed to identify disease-causing mutations in these regions. However, mutations affecting several other genes have been reported in cases of rare PDB-like syndromes. For example, loss of function mutations have been reported in TNFRSF11B gene causing juvenile PDB, a rare early-onset form of PDB. ${ }^{18,19}$ Certain missense mutations in the VCP gene cause Inclusion Body Myopathy Paget's disease and frontotemporal dementia, ${ }^{20}$ and mutations in hnRNPA2B1and hnRNPA1 were recently reported to cause a rare syndrome characterised by dementia, myopathy, PDB and amyotrophic lateral sclerosis. ${ }^{21}$ In addition, mutations in the TNFRSF11A gene cause three related PDB-like syndromes: expansile skeletal hyperphosphatasia, familial expansile osteolysis and early-onset PDB. ${ }^{22-24}$

\section{Genes and Genetic Loci Predisposing to PDB}

Genome-wide association studies (GWAS) have resulted in the identification of seven genetic loci that predispose to PDB. ${ }^{25,26}$ The variants identified were confirmed in several independent populations, providing strong evidence for their involvement in PDB susceptibility. These include susceptibility loci on chromosome 1, 7, 8, 10,14, 15 and 18 and are discussed below. It is interesting to note that the frequency of the risk allele for loci located on chromosome 1, 7, 8 and 15 is substantially higher in the European population compared with that observed in the Asian or African population, which may partially explain the variations in disease prevalence between ethnic groups.

\section{Chromosome 1p13 (CSF1)}

Common genetic variants at the $1 \mathrm{p} 13$ locus were associated with PDB risk in GWAS of familial and sporadic PDB. ${ }^{25,26}$ The associated region from this locus extends over approximately 120 kilo bases $(\mathrm{kb})$ and contains only one gene (CSF1), which encodes macrophage-colony stimulating factor (M-CSF). The CSF1 gene is a strong candidate for PDB susceptibility, as it has a critical role in osteoclast formation and survival. ${ }^{27}$ When M-CSF binds to c-FMS receptor, a signalling pathway is initiated leading to the activation of ERK and AKT resulting in the expression of genes that promote the proliferation and survival of osteoclast precursors. In addition, clinical studies have shown that PDB patients have increased serum levels of $\mathrm{M}-\mathrm{CSF}^{28}$ providing strong evidence for the involvement of CSF1 in the pathogenesis of PDB. The associated single-nucleotide polymorphisms (SNPs) from this region are located upstream CSF1, and carriers of the risk allele have $\sim 70 \%$ increased risk of developing PDB. ${ }^{25}$ The functional variants that predispose to PDB at this locus remain to be identified, but two of the PDB-associated SNPs are located in a region that is rich with histone 3 lysine 27 acetylation mark (H3K27Ac) containing numerous transcription factor binding sites. This suggests that these SNPs could predispose to PDB by influencing CSF1 gene expression, leading to enhanced osteoclast formation, but further functional studies will be required to investigate this possibility.

\section{Chromosome 7q33}

Several SNPs on 7q33 showed strong evidence for association with PDB susceptibility in an extended GWAS. ${ }^{26}$ The associated region is bounded by two recombination hotspots and contains three known genes (CNOT4, NUP205 and $S L C 13 A 4)$. Although any of these genes could be responsible for the association with PDB due to extensive linkage disequilibrium in the region, the strongest signal was located within the NUP205 gene. This gene encodes a $205 \mathrm{kD}$ nucleoporin protein that forms part of the nuclear pore complex, which has a role in the regulation of transport between the cytoplasm and the nucleus. None of the genes located within this region are known to have a role in bone metabolism, and thus further work will be required to investigate the mechanism by which variants at this locus predispose to PDB.

\section{Chromosome 8 (DCSTAMP)}

A genomic region that spans $\sim 220 \mathrm{~kb}$ on chromosome $8 \mathrm{q} 22$ was found to be associated with $\mathrm{PDB} .{ }^{26}$ The strongest association signals clustered within an 18-kb Linkage disequilibrium block spanning the entire dendritic cells-specific transmembrane protein (DCSTAMP; previously known as TM7SF4). This is a strong functional candidate gene for PDB, as the expression of DCSTAMP is upregulated by RANKL in osteoclast precursors ${ }^{29}$ where it is required for the fusion of osteoclast precursors to form mature osteoclasts. ${ }^{30}$ Details of signalling partners for DCSTAMP are not well known, but a study has shown that the connective tissue growth factor CCN2 binds DCSTAMP resulting in stimulation of osteoclast fusion. ${ }^{31}$ The mechanisms by which variants in DCSTAMP predispose to PDB are unknown, but the SNP with the strongest association with PDB risk in GWAS is also an expression quantitative trait locus that was found to regulate the expression of DCSTAMP in peripheral blood monocytes. ${ }^{32}$ It seems likely that variants at this locus contribute to PDB susceptibility by upregulating DCSTAMP expression leading to enhanced osteoclast fusion.

\section{Chromosome 10p13 (OPTN)}

A common genetic variant ( $r$ 1561570) on 10p13 within the OPTN gene was found to increase the risk of PDB by $\sim 60 \%$ in a GWAS of familial and sporadic PDB. ${ }^{25}$ This genomic region has been previously linked to familial PDB, but the predisposing gene has not been identified. ${ }^{11}$ OPTN encodes optineurin, which is a widely expressed cytoplasmic protein with multiple cellular functions. Optineurin contains an ubiquitin binding domain similar to that present in NEMO (a component of IKK complex involved in the NFKB signalling pathway; Figure 1). Studies have shown that optineurin negatively regulates tumour necrosis factor- $\alpha$-induced $N F \kappa B$ activation in immune cells by competing with NEMO for ubiquitylated RIP. ${ }^{33}$ The role of OPTN in bone metabolism was unknown, but studies in our lab have recently shown that OPTN has a role in regulating bone turnover. ${ }^{34}$ The PDB-associated SNP rs1561570 was found to regulate OPTN gene expression in peripheral blood monocytes $^{34}$ so that the risk allele was associated with reduced OPTN expression. Furthermore, Knockdown of optineurin in bone marrow-derived macrophages leads to enhanced osteoclast differentiation and hypernucleation. Mice with a loss of function mutation in the OPTN gene exhibit enhanced bone turnover with increased sensitivity of osteoclast precursors to RANKL stimulation. In addition, OPTN negatively regulates RANKL-induced NF $\kappa B$ activation in osteoclast precursors by a mechanism that requires ubiquitin binding and involves interaction with CYLD. ${ }^{34}$ We have also found that OPTN inhibits 
osteoclast differentiation by another mechanism that involves induction of interferon- $\beta$, which is known to inhibit osteoclast differentiation. ${ }^{35}$ These findings indicate that common genetic variant at the OPTN locus increases susceptibility to PDB by reducing levels of OPTN expression leading to enhanced osteoclast differentiation and that OPTN acts as a negative regulator of osteoclast differentiation by a complex mechanism that involves modulation of $\mathrm{NF} \kappa \mathrm{B}$ and interferon- $\beta$ signalling pathways. $^{34}$

\section{Chromosome 14q32 (RIN3)}

The chromosome $14 q 32$ region was identified as a candidate locus for PDB by GWAS, which showed an association with SNP that lay within a $62 \mathrm{~kb}$ genomic region. ${ }^{26}$ The associated region is bounded by two recombination hotspots and contains only one known gene (RIN3), which encodes the Ras and the Rab interactor 3 . This protein has a role in vesicular trafficking through interaction with small GTPases such as Ras and Rab. ${ }^{36}$ We have fine mapped this region in a recent study using targeted DNA sequencing and found that the association with PDB was driven by a common missense variant in RIN3 (p.R279C) that was in strong linkage disequilibrium with the top GWAS hit (rs10498635). An additional 13 rare missense variants were identified in this study, of which seven were novel and detected only in PDB cases. These rare variants, when combined, showed strong association with the disease. ${ }^{37}$ The p.R279C and most of the rare variants were clustered in a region that encodes a proline-rich domain of the protein, and many of these variants were predicted to be pathogenic. ${ }^{37}$ The function of RIN3 in bone metabolism is currently unknown, but we have recently shown that $R I N 3$ is expressed in bone tissue and its expression level was $\sim 10$-fold higher in osteoclasts compared with osteoblasts. ${ }^{37}$ The susceptibility to PDB at this locus seems to be mediated by a combination of common and rare missense coding variants in RIN3, which appear to influence PDB susceptibility by affecting the osteoclast function.

\section{Chromosome 15q24}

A genomic region spanning approximately $200 \mathrm{~kb}$ on chromosome $15 q 24$ was associated with PDB risk. ${ }^{26}$ The associated region contains two genes (promyelocytic leukaemia (PML) and GOLGA6), but SNPs with the highest association signal were clustered within the $P M L$ and the strongest signal was observed for a SNP, which results in a phenylalanine to leucine amino acid change at codon 645 (F645L) of the PML protein. $\mathrm{PML}$ is involved in the pathogenesis of promyelocytic leukaemia, but its function in bone metabolism is unclear. It has been shown that $\mathrm{PML}$ is involved in a wide range of cellular processes including apoptosis, tumour suppression, regulation of cell division, differentiation of myeloid precursor cells and transforming growth factor- $\beta$ (TGF- $\beta$ ) signalling. Previous studies have shown that cells from $\mathrm{pml}$ knock out mice are resistant to TGF- $\beta$-dependent growth arrest and apoptosis, had impaired induction of TGF- $\beta$ target genes and exhibited abnormal nuclear translocation of the TGF- $\beta$ signalling proteins Smad2 and Smad3. ${ }^{38}$ As TGF- $\beta$ is known to have a role in the regulation of bone remodelling, it is possible that the association between PDB and PML could be mediated by an effect on TGF- $\beta$ signalling, but further research will be required to investigate this possibility. The inhibitory effect of PML on apoptosis is another possible mechanism by which this gene could contribute to PDB in view of the findings that cultured osteoclasts from PDB patients have reduced apoptosis compared with cells from controls. ${ }^{39}$

The GOLGA6A gene is also located in the associated region and encodes a protein that belongs to golgin, a family of coiledcoil proteins associated with the Golgi apparatus and have a role in membrane fusion and as structural supports. The function of GOLGA6A in bone metabolism has not been determined, but mutations in other members of the golgin family have been shown to cause a lethal skeletal dysplasia and a severe form of osteoporosis. ${ }^{40,41}$ Further studies will be required to identify the genetic variants responsible for the association with PDB.

\section{Chromosome 18q21 (TNFRSF11A)}

TNFRSF11A is located on chromosome 18q21 and encodes receptor activator of NFKB (RANK). Several SNPs at the TNFRSF11A locus were associated with PDB risk in a candidate gene association study of PDB patients from Belgium, UK and the Netherlands. ${ }^{42}$ Two common SNPs located downstream TNFRSF11A were associated with 50\% increased risk of PDB in a large GWAS. ${ }^{25}$ RANK has long been known to have a critical role in osteoclast differentiation and function. Stimulation of RANK with RANKL leads to the activation of the NF $\kappa B$ signalling pathway and increased expression of osteoclast-specific genes (Figure 1). Mice with targeted disruption of TNFRSF11A exhibit severe osteopetrosis due to complete absence of osteoclasts and loss of function mutations in TNFRSF11A cause osteoclast-poor osteopetrosis in humans. Mutations affecting the signal peptide region of RANK cause the PDB-like syndromes of Familial Expansile osteolysis, early-onset familial PDB and expansile skeletal hyperphosphatasia. ${ }^{22-24}$ These syndromes, although rare, share many clinical and radiographic features with the classical form of PDB, but they generally have earlier age of onset and more extensive phenotype. All these syndromes are caused by mutations affecting the first exon of TNFRSF11A, which are predicted to interfere with cleavage of the signal peptide and impaired receptor function, but the exact mechanisms how this lead to focal osteolytic lesions remain to be investigated. Although TNFRSF11A is located in a region of chromosome 18q22, which has been linked to PDB in some families, ${ }^{43}$ mutations of TNFRSF11A have not so far been identified in patients with classical PDB. ${ }^{44}$ One mechanism that could explain the association with PDB risk reported from the GWAS study is that common SNPs in the TNFRSF11A could affect gene expression level, leading to increased $N F \kappa B$ activation and increased osteoclast activity. Further evidence of the involvement of common SNPs in the TNFRSF11A in bone metabolism is provided from GWAS studies in osteoporosis, in which the same SNP (rs3018362) that showed association with $\mathrm{PDB}$ risk was also associated with reduced bone mineral density (BMD). ${ }^{45}$

\section{Other PDB-susceptibility Loci}

Previous studies have reported many other genetic loci associated with PDB susceptibility, but the evidence was based on isolated findings without robust replication in subsequent studies. A locus on chromosome $5 q 31$ showed strong evidence for linkage to PDB in French-Canadian population, ${ }^{5}$ but this locus was not replicated in subsequent linkage or association studies in other populations. Common genetic variants within 
TNFRSF11B have been associated with PDB risk in women, ${ }^{46}$ but this finding was not replicated in GWAS of PDB. ${ }^{26}$ The TNFRSF11B gene encodes osteoprotegerin, which has an important role in the RANK signalling pathway, by acting as a decoy receptor for RANKL, resulting in the inhibition of osteoclastogenesis (Figure 1). Other genomic loci and candidate genes have been reported to be linked or associated with PDB, but the evidence was suggestive rather than statistically significant or based on a small sample size, and it is likely that these findings are false positive.

\section{Genetic Factors and Disease Severity}

The clinical presentation and extent of complications in PDB vary widely between patients. Although this variation in disease severity can be attributed to environmental triggers and/or genetic factors, studies on the contribution of environmental factors are limited. However, genetic factors have been shown to contribute to the severity of PDB. Carriers of SQSTM1 mutations tend to have more extensive PDB than patients without mutations, and disease complications are also more common. ${ }^{47}$ Common genetic variants from the genetic loci that were identified by GWAS are also associated with disease extent and severity of complications in a recent meta-analysis involving subjects from Italy, UK, Spain and Western Australia. ${ }^{48}$ In this study, the disease extent was defined by the number of affected bones, and severity was assessed by devising a composite disease severity score based on clinical features and complications (such as number of affected bone, bone deformity, previous orthopaedic surgery for PDB and previous bisphosphonate treatment). For the seven genetic loci, a risk allele score was calculated for each patient based on the number of risk alleles carried from the seven genetic loci identified by GWAS. A strong association was found between risk allele scores and disease severity in SQSTM1-negative subjects. Patients carrying high number of risk allele scores tend to have more extensive disease and suffer from more severe complications when compared with patients carrying small number of risk alleles (Figure 2). The association of risk allele scores with disease extent and severity remained statistically significant in this study after inclusion of SQSTM1positive patients, but the effect size of SQSTM1 mutations was approximately three times that of risk allele scores. Patients carrying both SQSTM1 mutations and high number of risk allele scores have the highest risk of developing severe disease compared with those who are SQSTM1 negative and carried small number of risk allele score.

\section{Genetic Factors and Clinical Implications}

Genetic studies of PDB have highlighted many new pathways that have not been previously implicated in the regulation of bone metabolism, resulting in major advancements in understanding of the pathophysiology of the disease. The genetic variants identified from the GWAS study showed surprisingly larger effect size on PDB risk compared with those observed in many common complex diseases. The risk of developing PDB was found to increase with the number of risk alleles carried so that patients in the top $10 \%$ of the risk allele score distribution have approximately 10 -fold increase in PDB risk compared with those in the bottom $10 \% .{ }^{26}$ Once the causal functional variants have been identified from each locus, it is likely that they would have a larger effect size on predicting both disease risk and severity making them a potentially useful tool in predicting disease risk and complication and in managing treatments. This would be particularly beneficial in PDB, which is often diagnosed when complications have already developed and irreversible skeletal damage has occurred.

\section{Genetic Factors and Focal Nature of PDB}

It has been suggested that environmental triggers, somatic mutations or epigenetic factors could explain why PDB is localised to specific bones. However, studies on the involvement of these factors in the focal nature of PDB are limited and provide no conclusive evidence to confirm or exclude their involvement. For example, a study has reported the occurrence of the P392L SQSTM1 somatic mutation in the affected bone of a small number of patients, ${ }^{49}$ and in a recent study this mutation was detected in peripheral blood monocytes of PDB patients. ${ }^{50}$ However, another study found no evidence for this mutation in the somatic tissue of 23 patients. ${ }^{51}$ Further studies are required to investigate whether somatic mutations or epigenetic changes contribute to disease localisation investigating the whole genome rather than targeting a single gene.

\section{Environmental Factors and PDB}

The focal nature of the disease along with epidemiological observations such as falling prevalence and incomplete penetrance suggest that environmental triggers may have a role in the disease pathophysiology. A recent study looking into the
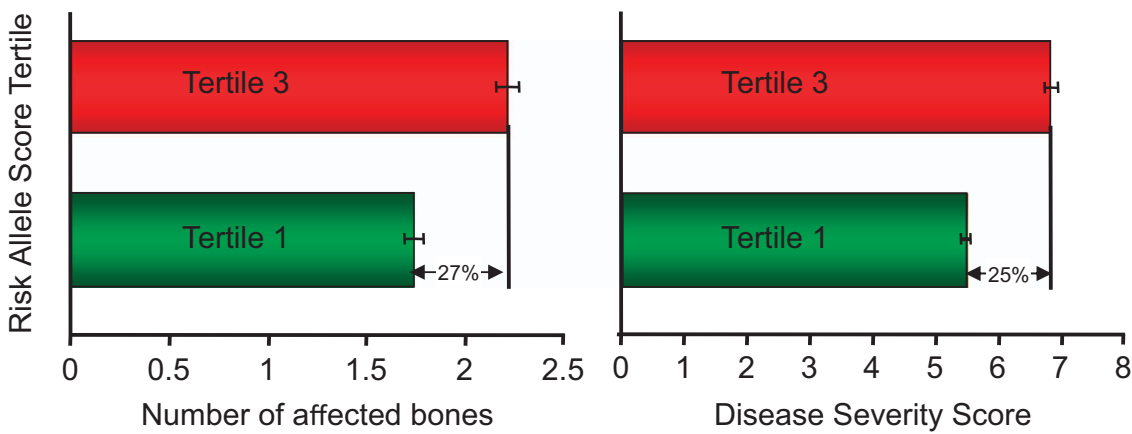

Figure 2 PDB-predisposing genetic variants and disease severity. Risk allele scores defined by the seven PDB susceptibility loci that were identified by GWAS in relation to disease extent and severity. Patients carrying the highest number of risk alleles (tertile 3) tend to have a $27 \%$ increase in disease extent and a $25 \%$ increase in disease severity compared with those carrying the lowest number of risk alleles (tertile 1). Data taken from Albagha et al. ${ }^{48}$ 
development and severity of PDB in first degree relatives of patients with SQSTM1-positive PDB showed evidence for gene-environment interaction. ${ }^{52}$ In this study, the development of PDB in offspring inheriting SQSTM1 mutations is delayed by a decade, and the disease is substantially less extensive compared with their affected parents. However, the nature of environmental factors causing this change in disease prevalence and severity is still unknown, but many factors have been suggested. Viral infection was the first suggested environmental trigger of PDB based on the observation of inclusion bodies in osteoclasts from affected patients. In fact, it was believed that PDB is caused by slow paramyxovirus infection, and these inclusion bodies were thought to be viral nucleocapsids. However, subsequent studies into the role of viral infection in the disease causation were inconclusive with some studies reporting evidence for virus transcripts in PDB patients, whereas other studies found no such evidence. ${ }^{53,54}$ Experimental studies have shown that paramyxoviruses enhance osteoclast formation in vitro and that bone turnover was enhanced in mice overexpressing measles virus nucleocapsid protein in osteoclasts. ${ }^{55}$ However, these observed effects are not specific to paramyxoviruses, as other viral proteins such as HLTV1 Tax were found to induce bone turnover in mice. ${ }^{56}$ Other possible environmental triggers such as childhood dietary calcium intake, mechanical loading of the skeleton, pesticides and toxins have also been suggested, but the evidence was based on isolated reports and further studies will be required to elucidate their contribution to the disease process. ${ }^{57-59}$

\section{Summary}

PDB is a late-onset disease characterised by focal areas of abnormal bone turnover. Current knowledge indicates that the disease is caused by complex interaction of genetic and environmental factors. Rare mutations in SQSTM1 gene cause about $10 \%$ of PDB cases by affecting the osteoclast function, whereas small effect common genetic variants in at least seven genetic loci predispose to the disease via hitherto unknown mechanisms. These loci have additive effects on disease susceptibility and interact with SQSTM1 mutations to affect disease severity. Many of these loci harbour genes that have important function in osteoclast differentiation such as CSF1, DCSTAMP and TNFRSF11A. Other susceptibility loci have highlighted new molecular pathways that have not been previously implicated in regulation of bone metabolism such as OPTN. Epidemiological studies indicate that environmental triggers also have a key role in PDB and interact with genetic factors to influence development and severity of the disease; however, further studies are needed to identify these triggers.

\section{Conflict of Interest}

The author declares no conflict of interest.

\section{Acknowledgements}

OMEA is supported by a consolidator grant from European Research Council (311723-GENEPAD).

\section{References}

1. van Staa TP, Selby P, Leufkens HG, Lyles K, Sprafka JM, Cooper C. Incidence and natural history of Paget's disease of bone in England and Wales. J Bone Miner Res 2002; 17:465-471.

2. Cooper C, Harvey NC, Dennison EM, van Staa TP. Update on the epidemiology of Paget's disease of bone. J Bone Miner Res 2006; 21(Suppl 2): 3-8.

3. Morales-Piga AA, Rey-Rey JS, Corres-Gonzalez J, Garcia-Sagredo JM, Lopez-Abente G. Frequency and characteristics of familial aggregation of Paget's disease of bone. J Bone Miner Res 1995; 10: 663-670.

4. Hocking LJ, Herbert CA, Nicholls RK, Williams F, Bennett ST, Cundy T et al. Genomewide search in familial paget disease of bone shows evidence of genetic heterogeneity with candidate loci on chromosomes 2q36, 10p13, and 5q35. Am J Hum Genet 2001; 69: 1055-1061.

5. Laurin N, Brown JP, Lemainque A, Duchesne A, Huot D, Lacourciere Y et al. Paget disease of bone: mapping of two loci at 5q35-qter and 5q31. Am J Hum Genet 2001; 69: 528-543.

6. Morissette J, Laurin N, Brown JP. Sequestosome 1: Mutation Frequencies, Haplotypes, and Phenotypes in Familial Paget's Disease of Bone. J Bone Miner Res 2006; 21(Suppl 2): 38-44.

7. Siris ES, Ottman R, Flaster E, Kelsey JL. Familial aggregation of Paget's disease of bone. J Bone Miner Res 1991; 6: 495-500.

8. Barker DJ. The epidemiology of Paget's disease of bone. [Review]. Br Med Bull 1984; 40: 396-400.

9. Mays S. Archaeological skeletons support a northwest European origin for Paget's disease of bone. J Bone Miner Res 2010; 25: 1839-1841.

10. Lucas GJ, Hocking LJ, Daroszewska A, Cundy T, Nicholson GC, Walsh JP et al. Ubiquitinassociated domain mutations of SQSTM1 in Paget's disease of bone: evidence for a founder effect in patients of British descent. J Bone Miner Res 2005; 20: 227-231.

11. Lucas G, Riches P, Hocking L, Cundy T, Nicholson G, Walsh J et al. Identification of a major locus for paget disease on chromosome 10p13 in families of british descent. J Bone Miner Res 2008; 23: 58-63.

12. Laurin N, Brown JP, Morissette J, Raymond V. Recurrent mutation of the gene encoding sequestosome 1 (SQSTM1/p62) in Paget disease of bone. Am J Hum Genet 2002; 70: $1582-1588$.

13. Hocking LJ, Lucas GJA, Daroszewska A, Mangion J, Olavesen M, Nicholson GC et al. Domain specific mutations in Sequestosome 1 (SQSTM1) cause familial and sporadic Paget's disease. Hum Mol Genet 2002; 11: 2735-2739.

14. Layfield R, Hocking LJ. SQSTM1 and Paget's disease of bone. Calcif Tissue Int 2004; 75 347-357.

15. Langston AL, Campbell MK, Fraser WD, MacLennan GS, Selby PL, Ralston SH. Randomised trial of intensive bisphosphonate treatment versus symptomatic management in paget's disease of bone. J Bone Miner Res 2010; 25: 20-31.

16. Daroszewska A, van't Hof RJ, Rojas JA, Layfield R, Landao-Basonga $E$, Rose $L$ et al. A point mutation in the ubiquitin-associated domain of SQSMT1 is sufficient to cause a Paget's disease-like disorder in mice. Hum Mol Genet 2011; 20: 2734-2744.

17. Kurihara N, Hiruma Y, Zhou H, Subler MA, Dempster DW, Singer FR et al. Mutation of the sequestosome 1 (p62) gene increases osteoclastogenesis but does not induce Paget disease. $J$ Clin Invest 2007; 117: 133-142.

18. Whyte MP, Obrecht SE, Finnegan PM, Jones JL, Podgornik MN, McAlister WH et al. Osteoprotegerin deficiency and juvenile Paget's disease. N Engl J Med 2002; 347: 175-184.

19. Chong B, Hegde M, Fawkner M, Simonet S, Cassinelli H, Coker M et al. Idiopathic hyperphosphatasia and TNFRSF11B mutations: relationships between phenotype and genotype. J Bone Miner Res 2003; 18: 2095-2104.

20. Watts GD, Wymer J, Kovach MJ, Mehta SG, Mumm S, Darvish D et al. Inclusion body myopathy associated with Paget disease of bone and frontotemporal dementia is caused by mutant valosin-containing protein. Nat Genet 2004; 36: 377-381.

21. Kim HJ, Kim NC, Wang YD, Scarborough EA, Moore J, Diaz Z et al. Mutations in prion-like domains in hnRNPA2B1 and hnRNPA1 cause multisystem proteinopathy and ALS. Nature 2013; 495: 467-473.

22. Hughes AE, Ralston SH, Marken J, Bell C, MacPherson H, Wallace RG et al. Mutations in TNFRSF11A, affecting the signal peptide of RANK, cause familial expansile osteolysis. Nat Genet 2000; 24: 45-48.

23. Whyte MP, Hughes AE. Expansile skeletal hyperphosphatasia is caused by a 15-base pair tandem duplication in TNFRSF11A encoding RANK and is allelic to familial expansile osteolysis. J Bone Miner Res 2002; 17: 26-29.

24. Nakatsuka K, Nishizawa Y, Ralston SH. Phenotypic characterization of early onset Paget's disease of bone caused by a 27-bp duplication in the TNFRSF11A gene. J Bone Miner Res 2003; 18: 1381-1385.

25. Albagha OM, Visconti MR, Alonso N, Langston AL, Cundy T, Dargie R et al. Genome-wide association study identifies variants at CSF1, OPTN and TNFRSF11A as genetic risk factors for Paget's disease of bone. Nat Genet 2010; 42: 520-524.

26. Albagha OM, Wani SE, Visconti MR, Alonso N, Goodman K, Brandi ML et al. Genome-wide association identifies three new susceptibility loci for Paget's disease of bone. Nat Genet 2011; 43: $685-689$

27. Tsurukai T, Udagawa N, Matsuzaki K, Takahashi N, Suda T. Roles of macrophage-colony stimulating factor and osteoclast differentiation factor in osteoclastogenesis. J Bone Miner Metab 2000; 18: 177-184.

28. Neale SD, Schulze E, Smith R, Athanasou NA. The influence of serum cytokines and growth factors on osteoclast formation in Paget's disease. QJM 2002; 95: 233-240.

29. Kukita T, Wada N, Kukita A, Kakimoto T, Sandra F, Toh K et al. RANKL-induced DC-STAMP is essential for osteoclastogenesis. J Exp Med 2004; 200: 941-946. 
30. Yagi M, Miyamoto T, Sawatani Y, Iwamoto K, Hosogane N, Fujita N et al. DC-STAMP is essential for cell-cell fusion in osteoclasts and foreign body giant cells. J Exp Med 2005; 202: 345-351.

31. Nishida T, Emura K, Kubota S, Lyons KM, Takigawa M. CCN family 2/connective tissue growth factor (CCN2/CTGF) promotes osteoclastogenesis via induction of and interaction with dendritic cell-specific transmembrane protein (DC-STAMP). J Bone Miner Res 2011; 26: 351-363.

32. Zeller T, Wild P, Szymczak S, Rotival M, Schillert A, Castagne R et al. Genetics and beyond--the transcriptome of human monocytes and disease susceptibility. PLOS ONE2010; 5: e10693.

33. Zhu G, Wu CJ, Zhao Y, Ashwell JD. Optineurin negatively regulates TNFalpha- induced NF-kappaB activation by competing with NEMO for ubiquitinated RIP. Curr Biol 2007; 17: 1438-1443.

34. Obaid R, Wani S, Azfer A, Hurd T, Jones R, Cohen $\mathrm{P}$ et al. Optineurin negatively regulates osteoclast differentiation by modulating $\mathrm{NF} \kappa \mathrm{B}$ and interferon signalling; implication for Paget's disease. Cell Rep (doi:10.1016/j.celrep.2015.09.071; in press).

35. Takayanagi H, Kim S, Matsuo K, Suzuki H, Suzuki T, Sato $\mathrm{K}$ et al. RANKL maintains bone homeostasis through c-Fos-dependent induction of interferon-beta. Nature 2002; 416: 744-749.

36. Coxon FP, Rogers MJ. The role of prenylated small GTP-binding proteins in the regulation of osteoclast function. Calcif Tissue Int 2003; 72: 80-84.

37. Vallet M, Soares DC, Wani S, Sophocleous A, Warner J, Salter DM et al. Targeted sequencing of the Paget's disease associated 14q32 locus identifies several missense coding variants in RIN3 that predispose to Paget's disease of bone. Hum Mol Genet 2015; 24: 3286-3295.

38. Lin HK, Bergmann S, Pandolfi PP. Cytoplasmic PML function in TGF-beta signalling. Nature 2004; 431: 205-211.

39. Chamoux E, Couture J, Bisson M, Morissette J, Brown JP, Roux S. The p62 P392L mutation linked to Paget's disease induces activation of human osteoclasts. Mol Endocrinol 2009; 23 : 1668-1680.

40. Smits $P$, Bolton $A D$, Funari $V$, Hong $M$, Boyden ED, Lu L et al. Lethal skeletal dysplasia in mice and humans lacking the golgin GMAP-210. N Engl J Med 2010; 362: 206-216.

41. Hennies HC, Kornak U, Zhang H, Egerer J, Zhang X, Seifert W et al. Gerodermia osteodysplastica is caused by mutations in SCYL1BP1, a Rab-6 interacting golgin. Nat Genet 2008; 40: 1410-1412.

42. Chung PYJ, Beyens G, Riches PL, Van Wesenbeeck L, de Freitas F, Jennes K et al. Genetic variation in the TNFRSF11A gene encoding RANK is associated with susceptibility to Paget's disease of bone. J Bone Miner Res 2010; 25: 2592-2605.

43. Hocking L, Slee F, Haslam SI, Cundy T, Nicholson G, Van HW et al. Familial Paget's disease of bone: patterns of inheritance and frequency of linkage to chromosome 18q. Bone 2000; 26: $577-580$.

44. Wuyts W, Van Wesenbeeck L, Morales-Piga A, Ralston S, Hocking L, Vanhoenacker F et al. Evaluation of the role of RANK and OPG genes in Paget's disease of bone. Bone 2001; 28: 104-107.
45. Rivadeneira F, Styrkarsdottir U, Estrada K, Halldorsson BV, Hsu YH, Richards JB et al. Twenty bone-mineral-density loci identified by large-scale meta-analysis of genome-wide association studies. Nat Genet 2009; 41: 1119-1206.

46. Beyens $G$, Daroszewska A, de Freitas $F$, Fransen $E$, Vanhoenacker $F$, Verbruggen $L$ et al Identification of sex-specific associations between polymorphisms of the osteoprotegerin gene, TNFRSF11B, and Paget's disease of bone. J Bone Miner Res 2007; 22 1062-1071.

47. Visconti MR, Langston AL, Alonso N, Goodman K, Selby PL, Fraser WD et al. Mutations of SQSTM1 are associated with severity and clinical outcome in Paget's disease of bone. J Bone Miner Res 2010; 25: 2368-2373.

48. Albagha OM, Visconti MR, Alonso N, Wani S, Goodman K, Fraser WD et al. Common susceptibility alleles and SQSTM1 mutations predict disease extent and severity in a multinational study of patients with Paget's disease. J Bone Miner Res 2013; 28 : 2338-2346.

49. Merchant A, Smielewska M, Patel N, Akunowicz JD, Saria EA, Delaney JD et al. Somatic mutations in SQSTM1 detected in affected tissues from patients with sporadic Paget's disease of bone. J Bone Miner Res 2009; 24: 484-494.

50. Guay-Belanger S, Picard S, Gagnon E, Morissette J, Siris ES, Orcel P et al. Detection of SQSTM1/P392L post-zygotic mutations in Paget's disease of bone. Hum Genet 2015; 134 53-65.

51. Matthews BG, Naot D, Bava U, Callon KE, Pitto RP, McCowan SA et al. Absence of somatic SQSTM1 mutations in Paget's disease of bone. J Clin Endocrinol Metab 2009; 94: 691-694.

52. Cundy T, Rutland MD, Naot D, Bolland M. Evolution of Paget's disease of bone in adults inheriting SQSTM1 mutations. Clin Endocrinol (Oxf) 2015; 83: 315-319.

53. Friedrichs WE, Reddy SV, Bruder JM, Cundy T, Cornish J, Singer FR et al. Sequence analysis of measles virus nucleocapsid transcripts in patients with Paget's disease. J Bone Miner Res 2002; 17: 145-151.

54. Ralston SH, Afzal MA, Helfrich MH, Fraser WD, Gallagher JA, Mee A et al. Multicenter blinded analysis of RT-PCR detection methods for paramyxoviruses in relation to Paget's disease of bone. J Bone Miner Res 2007; 22: 569-577.

55. Kurihara N, Reddy SV, Menaa C, Anderson D, Roodman GD. Osteoclasts expressing the measles virus nucleocapsid gene display a pagetic phenotype. J Clin Invest 2000; 105: $607-614$.

56. Ruddle NH, Li CB, Horne WC, Santiago P, Troiano N, Jay G et al. Mice transgenic for HTLVLTR-tax exhibit tax expression in bone, skeletal alterations, and high bone turnover. Virology 1993; 197: 196-204.

57. Siris ES. Epidemiological aspects of Paget's disease: family history and relationship to other medical conditions. Semin Arth Rheum 1994; 23: 222-225.

58. Barker DJ, Gardner MJ. Distribution of Paget's diease in England, Wales and Scotland and a possible relationship with vitamin D deficiency in childhood. Br J Prev Soc Med 1974; 28 : 226-232.

59. Solomon LR. Billiard-player's fingers: an unusual case of Paget's disease of bone. Br Med J 1979; 1: 931 